\title{
Simultaneous Localization and Odometry Calibration for Mobile Robot
}

\author{
Agostino Martinelli, Nicola Tomatis, Adriana Tapus and Roland Siegwart \\ Autonomous Systems Lab Swiss Federal Institute of Technology Lausanne (EPFL) \\ EPFL, CH-1015 Lausanne, Switzerland e-mail: agostino.martinelli, nicola.tomatis, \\ adriana.tapus, roland.siegwart@epfl.ch*
}

\begin{abstract}
This paper presents both the theory and the first experimental results of a new method which allows simultaneously estimating of the robot configuration and the odometry error (both systematic and non-systematic) during the mobile robot navigation. The estimation of the non-systematic components is carried out through an augmented Kalman filter which estimates a state containing the robot configuration and the parameters characterizing the systematic component of the odometry error. It uses encoder readings as inputs and the readings from a laser range finder as observations. The estimation of the non-systematic component is carried out through another Kalman filter where the observations are obtained by two subsequent robot configurations provided by the previous augmented Kalman filter.
\end{abstract}

Key Words: Robot Navigation, Kalman filter, Odometry Learning

\section{Introduction}

Determining the odometry errors of a mobile robot is very important both in order to reduce them, and to know the accuracy of the state configuration estimated by using encoder data.

Odometry errors can be both systematic and nonsystematic. In a series of papers Borenstein and collaborators $[3,4,5,6,7,8,19]$ investigated on possible sources of both kind of errors. A review of all the types of these sources is given in [8]. In the work by Borenstein and Feng [7], a calibration technique called UMBmark test has been developed to calibrate for systematic errors of a mobile robot with a differential drive. Larsen et al. [11, 12] suggested an algorithm that uses the robot's sensors to automatically calibrate the robot as it operates. In particular,

\footnotetext{
*This work has been supported by the European project RECSYS (Real-Time Embedded Control of Mobile Systems with Distributed Sensing)
}

they introduced an augmented Kalman filter $(A K F)$ which simultaneously estimates the robot configuration and the parameters characterizing the systematic odometry error. This filter uses encoder readings as inputs and vision measurements as observations. They referred to a mobile robot with a differential drive system.

Many investigations have been carried out on the odometry error from a theoretical point of view. Wang [18] and Chong and Kleeman [9] analyzed the non-systematic errors and computed the odometry covariance matrix $Q$ for special kind of the robot trajectory. Kelly [10] presented the general solution for linearized systematic error propagation for any trajectory and any error model. Martinelli [14] derived general formulas for the covariance matrix and also suggested a strategy to estimate the model parameters for a mobile robot with a synchronous drive system. This strategy is based on the evaluation of the mean values of some quantities (called observables) which depend on the model parameters and on the chosen robot motion.

In a recent work Martinelli and Siegwart [16] suggested a method to estimate both systematic and non-systematic odometry error of a mobile robot, during navigation. Concerning the systematic component, they adopted the same $A K F$ introduced by Larsen et al. $[11,12]$ by considering also the case of a synchronous drive. Concerning the nonsystematic parameters, they introduced a new filter (the Observable Filter, $O F$ ) where the state to be estimated contains the parameters characterizing the non-systematic error and the observations are provided by the observables as defined in [14] and which can be evaluated by knowing two subsequent robot configurations.

In this paper the new method [16] is empirically validated by experimentation on a real differential drive robot. In section 2 we introduce the model here adopted to characterize the odometry error for 
a mobile robot with a differential drive. In section 3 we summarize the $A K F$ introduced by Larsen et al. $[11,12]$. The $O F$ is presented in section 4 and discussed for the specific case implemented in the experiments. In particular the influence on the accuracy on the non-systematic parameter estimations due to the error on the systematic error evaluation is deeply investigated. In section 5 we show and discuss the experimental results obtained with the fully autonomous robot Donald Duck. Finally, some conclusions are given in section 6

\section{The odometry error model}

A simple way to characterize the odometry error for a mobile robot with a differential drive system is obtained by modeling separately the error in the translation of each wheel [9]. The actual translation of the right/left wheel related to the $i^{\text {th }}$ time step is assumed to be a gaussian random variable satisfying the following relation:

$$
\begin{gathered}
\delta \rho_{i}^{R / L}=\overline{\delta \rho}_{i}^{R / L}+\nu_{i}^{R / L} \\
\overline{\delta \rho}_{i}^{R / L}=\delta \rho_{i}^{e R / L} \delta_{R / L} \\
\nu_{i}^{R / L} \sim N\left(0, K_{w}\left|\delta \rho_{i}^{e R / L}\right|\right)
\end{gathered}
$$

In other words, both $\delta \rho_{i}^{R}$ and $\delta \rho_{i}^{L}$ are assumed gaussian random variables, whose mean values are given by the encoder readings (respectively $\delta \rho_{i}^{e R}$ and $\delta \rho_{i}^{e L}$ ) corrected for the systematic errors (which are assumed to increase linearly with the distance traveled by each wheel), and whose variances also increase linearly with the traveled distance. Moreover, it is assumed that $\delta \rho_{i}^{R}$ and $\delta \rho_{i}^{L}$ are uncorrelated. With respect to the Chong-Kleeman model, only one parameter $\left(K_{w}\right)$ is here adopted to characterize both the variances for the right and left wheel. The robot translation and rotation are given by the following relations:

$$
\delta \rho_{i}=\frac{\delta \rho_{i}^{R}+\delta \rho_{i}^{R}}{2} \quad \delta \theta_{i}=\frac{\delta \rho_{i}^{R}-\delta \rho_{i}^{L}}{d \delta_{d}}
$$

where $d$ is the estimated distance between the wheels and $\delta_{d}$ characterizes the uncertainty on this estimation. Clearly, the robot translation and rotation are correlated accordingly to the equations (1-4). The odometry error model here proposed is based on 4 parameters. Three $\left(\delta_{R}, \delta_{L}\right.$ and $\left.\delta_{d}\right)$ characterize the systematic components whereas the last one $\left(K_{w}\right)$ characterizes the non-systematic components.
In section 3 and 4 we introduce the strategy to simultaneously estimate all these parameters during the robot navigation.

\section{Systematic Parameters Estimation during Navigation}

In order to estimate the parameters characterizing the systematic error we adopt the same $A K F$ introduced by Larsen et al. [11, 12]. This filter estimates a state (the augmented state) containing the robot configuration and the systematic parameters, through an extended Kalman filter $(E K F)$.

Let be $X$ the robot configuration $\left(X=[x, y, \theta]^{T}\right)$ and $X_{a}$ the augmented state. We have

$$
X_{a}=\left[x, y, \theta, \delta_{R}, \delta_{L}, \delta_{d}\right]^{T}
$$

The state $X$ evolves accordingly to the dynamical equation $X_{i+1}=f\left(X_{i}, U_{i}\right)$ where $U_{i}=\left[\delta \rho_{i}^{R}, \delta \rho_{i}^{L}\right]^{T}$. The observation at the $i^{\text {th }}$ time step depends on the current robot configuration and it is assumed to be affected by an error $w_{i}$ with a gaussian distribution, zero-mean and covariance matrix $\left.R_{i}=<w_{i} w_{i}^{T}\right\rangle$

$$
z_{i}=h\left(X_{i}, w_{i}\right)
$$

A simple example for this function is obtained by define $z$ as the vector containing all the distances in several directions of observation from the robot configuration towards the landmarks (e.g. straight lines stored in a map a priori known). In this case the function $h$ characterizes the measurement prediction of a laser range finder. In the experiments carried out in our lab and discussed in section 5, this function was not the previous one since, instead of using the raw data, we extracted the straight lines from the data (see also [1]).

The dynamical equation for the augmented state $X_{a}$ is given by the equation:

$$
X_{a i+1}=f_{a}\left(X_{a i}, U_{i}\right)
$$

The function $f_{a}$, restrictly to the first three components, is obtained directly from the function $f$ including the dependence on the systematic parameters in the input $U_{i}$; concerning the last three components $f_{a}$ is the identity function since there is no evolution in time for the error parameters.

In order to obtain the $E K F$ equations for the augmented state (i.e. the equations of the $A K F$ ), it is necessary to compute the Jacobian $F_{a}$ of the function $f_{a}$ with respect to $X_{a}$ and the Jacobian $G_{a}$ of the function $f_{a}$ with respect to the vector $\nu$, which is $\left[\nu^{R}, \nu^{L}\right]^{T}($ eq. $(3))$ : 


$$
F_{a}=\left.\nabla_{X_{a}} f_{a}\right|_{X_{a}(i \mid i), \bar{U}_{i}} \quad G_{a}=\left.\nabla_{\nu} f_{a}\right|_{X_{a}(i \mid i), \bar{U}_{i}}
$$

where $X_{a}(i \mid i)$ is the state estimated at the previous time step and $\bar{U}_{i}$ is the mean value of the vector $U_{i}$ previously defined. The computation of these matrix can be found in $[11,12]$.

\section{Non-Systematic Parameters Estima- tion during navigation}

The non-systematic parameter $K_{w}$ cannot be evaluated following the previous method. Indeed, by including $K_{w}$ in the augmented state, the Kalman gain related to this parameter is null.

The $O F$ suggested in [16] and here adopted is based on the observables defined in [14]. The observables are random variables related to a given robot motion whose statistical properties (mean value and variance) depend on the parameters characterizing the odometry error and on the robot trajectory in the odometry reference frame. Moreover, it is possible to evaluate the observable mean value only by knowing the actual initial and final configuration. These actual configurations are directly estimated by the $A K F$ of above. The $O F$ is an $E K F$ whose estimated state contains the non-systematic parameters (in this case only the parameter $K_{w}$ ). Since the environment is assumed to be homogeneous and stationary the dynamical equation of this filter $([2,13])$ is the identity:

$$
K_{w_{i+j}}=f_{K}\left(K_{w_{i}}\right)=K_{w_{i}}
$$

where we use $i+j$ instead of $i+1$ to remark that the frequency of this second filter is not necessarily the same of the previous one $(A K F)$.

The observational equation is the following:

$$
z_{i+j}^{O b s}=m^{O b s}\left(K_{w_{i+j}}\right)+w_{i+j}^{O b s}
$$

where $z^{O b s}$ is the observable mean value as estimated by the $A K F$, i.e. by knowing the robot configuration at the time step $i^{t h}$ and $(i+j)^{t h}, m^{O b s}\left(K_{w}\right)$ is the mean value of the observable analytically computed by knowing the trajectory in the odometry frame, and $w^{\text {Obs }}$ is a zero-mean random variable whose covariance matrix contains both the covariance matrix of the observable and the error on the robot configuration and on the systematic parameter estimation (given by the matrix $P_{a}(i \mid i)$ and $P_{a}(i+j \mid i+j)$ ), since the observable mean value is estimated from two subsequent robot configuration estimations obtained from the $A K F$ and these estimations are affected by an error given by the matrix $P_{a}$. Observe that $z^{O b s}$ is an estimation of the observable mean value obtained only through one realization of the robot motion (since only one realization is obviously available). For this reason it is very important to include in the covariance matrix of $w^{\text {Obs }}$ the covariance matrix of the observable.

Let $\Delta \theta_{a}$ and $\Delta \theta_{o}$ be the robot orientation change between the time $i+j$ and $i$, respectively estimated by the $A K F$ and the odometry corrected for the systematic errors by using the systematic parameters estimated by the $A K F$ at the $(i+j)^{t h}$. Finally, let $\Delta \theta$ be the actual orientation change. The observable we adopt here (which is the same adopted in [16] for the differential drive) is:

$$
z^{\text {Obs }}=\left(\Delta \theta_{a}-\Delta \theta_{o}\right)^{2}
$$

On the basis of the odometry error model introduced in section 2 we obtain for the mean value and variance of this observable [17] (we neglect the covariance between the error on the robot orientation obtained by the $A K F$ and the error on the orientation obtained with the odometry):

$$
\begin{gathered}
m^{O b s}\left(K_{w}\right)=\frac{K_{w} S}{b^{2}}+\sigma_{\theta}^{2}+\chi^{2} \\
\sigma_{O b s}^{2}=2 \times \\
\times\left[\sigma_{\theta}^{4}+\frac{K_{w}^{2} S^{2}}{b^{4}}+2 \chi^{2}\left(\sigma_{\theta}^{2}+\frac{K_{w} S}{b^{2}}\right)+2 \sigma_{\theta}^{2} \frac{K_{w} S}{b^{2}}\right]
\end{gathered}
$$

where:

- $S=s_{R}+s_{L}$, and $s_{R}$ and $s_{L}$ are the distances traveled respectively by the right and left wheel, between the time $i$ and $i+j$ as estimated by the odometry (i.e. $s_{R / L}=\sum_{k=i}^{i+j}\left|\delta \rho_{k}^{e R / L}\right|$ )

- $b$ is the actual distance between the wheel, namely $b=\delta_{d}^{t} d$, where we denote with $\delta_{R}^{t}, \delta_{L}^{t}$ and $\delta_{d}^{t}$ the actual (unknown) values of the systematic parameters

- $\sigma_{\theta}^{2}$ is the variance related to the robot orientation estimated by the $A K F$, i.e. $\sigma_{\theta}^{2}=$ $P_{a}(i \mid i)[3,3]+P_{a}(i+j \mid i+j)[3,3]$, since the orientation is the third element in the augmented state (eq.(5))

- $\chi$ takes into account the uncertainty on the systematic parameters and is explicitly given by the following expression:

$$
\begin{gathered}
\chi=\chi\left(s_{R}, s_{L}\right)= \\
=\frac{s_{R}\left(\delta_{d} \delta_{R}^{t}-\delta_{d}^{t} \delta_{R}\right)-s_{L}\left(\delta_{d} \delta_{L}^{t}-\delta_{d}^{t} \delta_{L}\right)}{b \delta_{d}}
\end{gathered}
$$


In order to estimate $K_{w}$ through the $O F$ it is necessary that the first term on the right side in the equation (11) is larger than the other two. In particular, for the term $\chi^{2}$ we do not have any estimation but we can only compute an over bound, obtaining:

$$
\chi^{2} \leq \frac{\epsilon^{2}}{\left(b \delta_{d}\right)^{2}}\left(\left|s_{R}-s_{L}\right|+S\right)^{2}=\chi_{M}^{2}
$$

where $\epsilon$ is the error on the systematic parameters (nearly the same value for all of them). As we expected, the error on the systematic parameters $\left(\chi^{2}\right)$ affects the observable mean value with the square of the traveled distance. On the other hand, the second term on the right side of the equation (11) is independent of the traveled distance. Finally, the first term containing $K_{w}$, depends linearly on the distance. Therefore, the best frequency for the $O F$ is fixed by requiring that the linear component is the largest. Clearly, as showed in Fig $1 b$ this requirement could not be satisfied (e.g. when the value of $K_{w}$ is very small). The value of $S_{0}$ showed in the figure corresponds to the $S$ where $\chi_{M}^{2}=\sigma_{\theta}^{2}$.

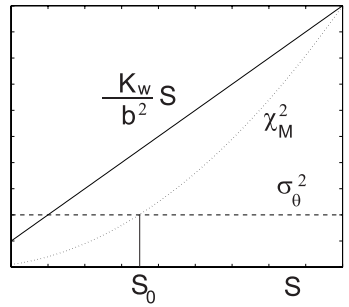

(a)

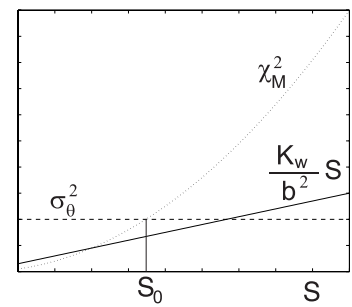

(b)
Figure 1: The three components appearing in the mean value expression (equation (11)) vs the distance traveled by the robot between two subsequent $O F$ updates. In the case showed in (b) it is not possible to estimate $K_{w}$ for any $S$.

In the next section we show the experimental results obtained by choosing the value of $S_{0}$ in order to fix the frequency of the $O F$.

\section{Results}

For the experiments, a fully autonomous mobile vehicle has been used. Donald Duck (figure 2) is a mobile robot with a differential drive. It is equipped with wheel encoders, a 360 laser range finder and a grey-level CCD camera (not used here). It is connected via radio ethernet only for data visualization via web and data logging for statistical purposes.

We performed two set of experiments. In each experiment the robot moved along a distance of about $300 \mathrm{~m}$ in our laboratory. The two set of experiments

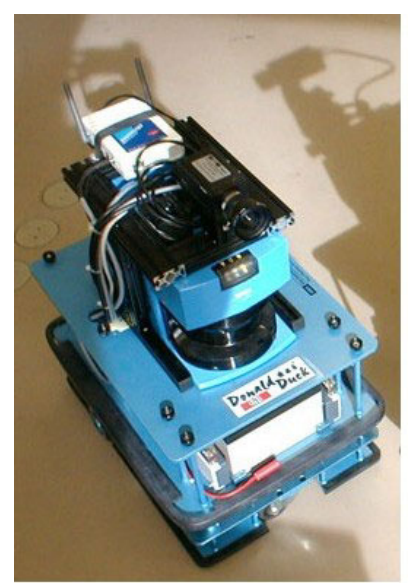

Figure 2: The autonomous robot Donald Duck. Its controller consists of a VME standard backplane with a Motorola PowerPC 604 microprocessor clocked at $300 \mathrm{Mhz}$. Among its peripheral devices, the most important are the wheel encoders, a 360 laser range finder and a grey-level CCD camera (not used here).

differed because in one case we added on both the robot wheels a small piece of tape in order to increase slightly the wheel diameters and to test the accuracy of the implemented $A K F$. In all the cases the $O F$ started to work only when the systematic parameter errors, as estimated by the covariance matrix of the $A K F\left(P_{a}\right)$, were smaller than $510^{-4}$. This accuracy was always achieved after about $100 \mathrm{~m}$.

Concerning the $A K F$, we set the initial covariance matrix $P_{a}$ as diagonal. Moreover, the variances corresponding to the systematic parameters were set equal to $(0.05)^{2}$ for all the three parameters. Finally, the initial values was fixed equal to 1.0 for all of them.

Regarding the non-systematic parameter $K_{w}$, we set in the most of the experiments, the initial value equal to $0.01 \mathrm{~m}$. This value is very large. Indeed, it corresponds to have a non-systematic error whose standard deviation after $100 \mathrm{~m}$ of navigation, is equal to $1 m$ for each wheel. Therefore, the $A K F$ at beginning used nearly only the laser range finder to localize the robot.

Figures 3, 4 and 5 concern the systematic parameter results. Dotted line is adopted for the case with the tape on the wheels. As expected, the values of $\delta_{R}$ and $\delta_{L}$ increase with respect to the case without tape. The variation is equal to about 0.01 corresponding to a diameter change of $0.4 \mathrm{~mm}$, since the wheel diameter is equal to $3.8 \mathrm{~cm}$. Fig. 5 shows also a change in the wheels base distance due to the tape. This change demonstrates that the point where the wheel touches the terrain is pushed out by the tape. 


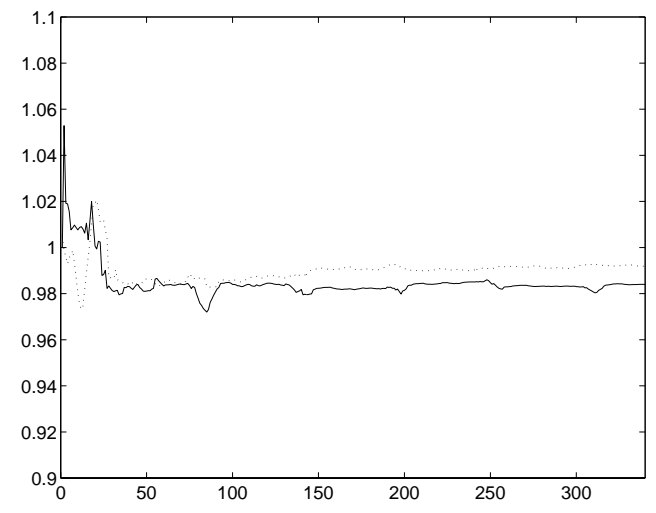

Figure 3: The $\delta_{R}$ parameter estimated by the AKF $v$ s the distance traveled by the robot (unity $m$ ). The dotted line refers to the case with the tape on the wheels

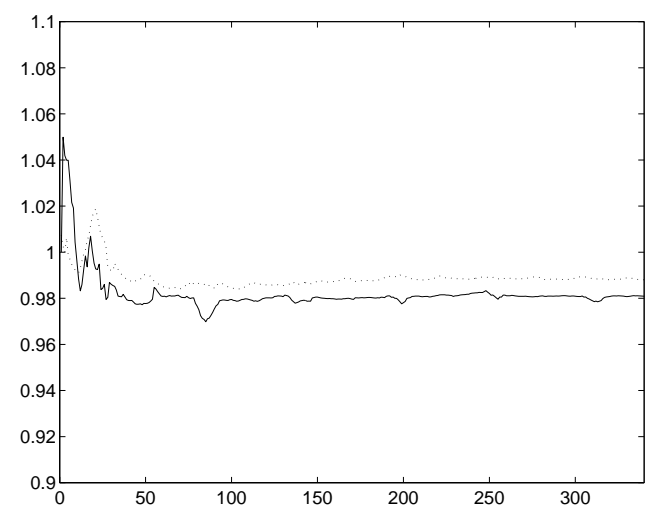

Figure 4: The $\delta_{L}$ parameter estimated by the AKF vs the distance traveled by the robot (unity $m$ ). The dotted line refers to the case with the tape on the wheels

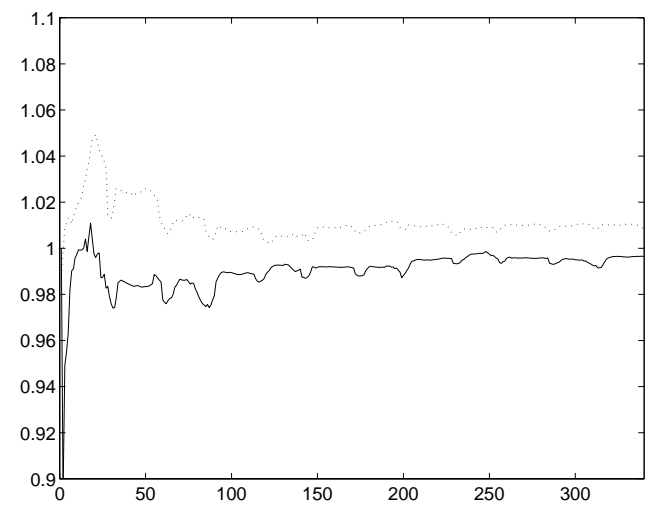

Figure 5: The $\delta_{d}$ parameter estimated by the AKF $v s$ the distance traveled by the robot (unity $m$ ). The dotted line refers to the case with the tape on the wheels

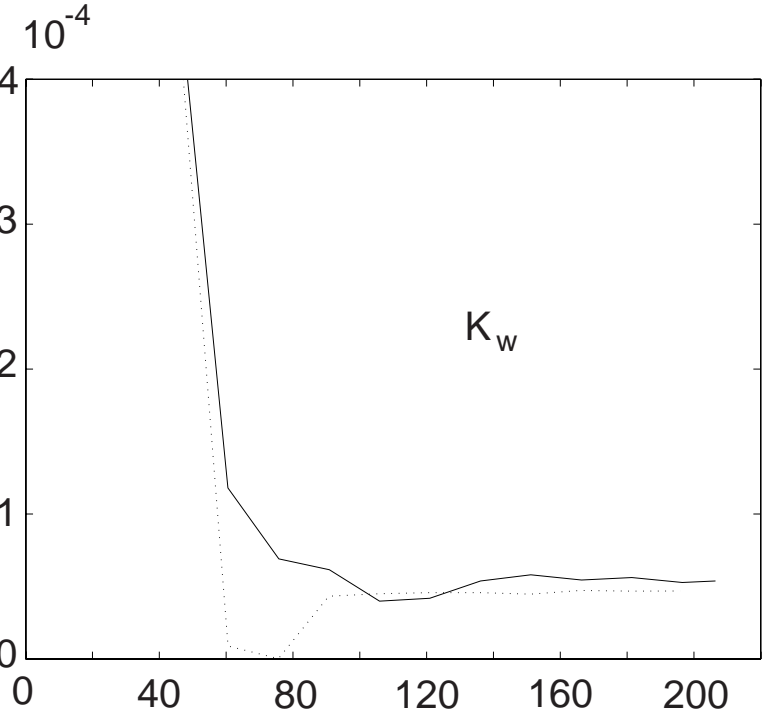

Figure 6: The non-systematic parameter $K_{w}$ as estimated by the $O F$ vs the distance traveled by the robot (unity $m$ in both axis). The dotted line refers to the case with the tape on the wheels

Fig. 6 concerns the non-systematic parameter results. Again, dotted line is adopted for the case with the tape on the wheels. In this case the tape does not produce variation. The frequency of the $O F$ was chosen accordingly to the considerations given in section 4. In particular, by imposing that the second and third term in the equation (11) are equal, we obtain from equation (14):

$$
S_{0} \simeq \frac{\sigma_{\theta} b}{\epsilon}
$$

which corresponds in our case to a value $S_{0} \simeq 15 \mathrm{~m}$ (the error in the orientation as estimated by the $A K F$, i.e. the second term in equation (11), is about always $\sigma_{\theta} \simeq 0.025 \mathrm{rad}, \epsilon \simeq 510^{-4}$ and $\left.b=0.3 \mathrm{~m}\right)$. Observe that the frequency of the $A K F$ is much higher $(\simeq 10 \mathrm{~cm})$. For this value of $S_{0}$ we obtain a value of the first term in the equation (11) about of ten times larger than the other two. This means that we are in the situation showed in fig. 1a. Similar results for the estimated $K_{w}$ were obtained by changing the value of $S_{0}$. In particular, we did many experiments in the range $10 m \leq S_{0} \leq 30 m$ obtaining a variation in $K_{w}$ within the $20 \%$. We also did other trials by changing the initial value of $K_{w}$ (always in the range $0.0001 m \leq K_{w} \leq 0.1 m$ ) obtaining again a slight variation in the results (within the 20\%). The final estimated $K_{w}$ showed in the fig. 6 are $K_{w}=(4.7 \pm 1.6) 10^{-5}$ and $K_{w}=(5.4 \pm 1.8) 10^{-5}$ respectively with and without tape. This value of $K_{w}$ corresponds to have a non-systematic error 
whose standard deviation after $100 \mathrm{~m}$ of navigation, is $\simeq 5 \mathrm{~cm}$ for each wheel.

\section{Conclusions}

A new filter, the Observable filter, was implemented for the estimation of the non-systematic odometry error during the robot navigation. This filter is based on the Observables (introduced in a previous work [14]) which provide the observations for an extended Kalman filter estimating a state containing the parameters characterizing the non-systematic odometry error. This new filter was used together with the augmented Kalman filter (introduced by Larsen et al. $[11,12])$ enabling the simultaneous estimation of the systematic and non-systematic odometry error during the robot navigation.

The experimental results show that it is possible to estimate the systematic error with high accuracy $(0.05 \%$ by moving the robot for $100 m)$ and the nonsystematic error with an accuracy of $30 \%$. Observe that our experiments were carried out in an indoor environment with a very smooth floor and therefore the non-systematic component is very low and very difficult to be evaluated.

We are performing some experiments showing the usefulness of an odometry autocalibration in the framework of the SLAM problem. We want to remark that in the localization problem with a precise map a priori known, and when a precise external sensor is available, the localization error is very small compared to the odometry error (calibrated or not), since the localization task is nearly completely relied to the external sensor. In the SLAM problem, however, the odometry could play a very important role especially in solving the data association problem.

\section{References}

[1] K.O.Arras, N.Tomatis, B.T.Jensen and R.Siegwart, "Multisensor on-the-fly localization: Precision and reliability for applications", Robotics and Autonomous Systems 34, pp. 131-143, 2001.

[2] Y.Bar-Shalom, T.E.Fortmann,, "Tracking and data association, mathematics in science and engineering", Vol 179, Academic Press, New York, 1988.

[3] Borenstein J., "Internal Correction of Dead-reckoning Errors with the Smart Encoder Trailer," International Conference on Intelligent Robots and Systems, vol. 1, pp. 127-134, 1994.

[4] Borenstein J., "The CLAPPER: A dual-drive Mobile Robot with Internal Correction of Dead-reckoning Errors," International Conference on Robotics and Automation, vol. 3, pp. 3085-3090, 1995.

[5] Borenstein J., Feng L., "Correction of systematic odometry errors in mobile robots ," International
Conference on Intelligent Robots and Systems, vol. 3, pp. 569-574, 1995.

[6] Borenstein J., Feng L., "Measurement and correction of systematic odometry errors in mobile robots," IEEE Transactions on Robotics and Automation, vol. 12, pp. 869-880, 1996.

[7] Borenstein J., Feng L., "UMBmark - A method for measuring, comparing and correcting dead-reckoning errors in mobile robots," Technical Report UMMEAM-94-22, University of Michigan.

[8] Borenstein J., "Experimental results from internal odometry error correction with the OmniMate mobile robot," IEEE Transactions on Robotics and Automation, vol. 14, pp. 963-969, 1998.

[9] Chong K.S., Kleeman L., "Accurate Odometry and Error Modelling for a Mobile Robot," International Conference on Robotics and Automation, vol. 4, pp. 2783-2788, 1997.

[10] Kelly A, "General Solution for Linearized Systematic Error Propagation in Vehicle Odometry," International Conference on Inteligent Robot and Systems (IROS01) Maui, Hawaii, USA, Oct. 29 - Nov. 3, pag 1938-1945, 2001

[11] T.D. Larsen, "Optimal Fusion of Sensors," PhD thesis, Department of Automation, Technical University of Denmark, Sept. 1998

[12] T.D. Larsen, M. Bak, N.A. Andersen and O. Ravn, "Location Estimation for Autonomously Guided Vehicle using an Augmented Kalman filter to Autocalibrate the Odometry," FUSION98 Spie Conference Las Vegas, USA, July 1998

[13] J.J. Leonard, H.F. Durrant-Whyte, "Directed Sonar Sensing for Mobile Robot Navigation," Kluwer Academic Publishers, Dordrecht, 1992.

[14] Martinelli A, "The odometry error of a mobile robot with a synchronous drive system", IEEE Trans. on Robotics and Automation Vol 18, NO. 3 June 2002, pp 399-405

[15] Martinelli A, "Evaluating the Odometry Error of a Mobile Robot," International Conference on Inteligent Robot and Systems (IROS02) Lausanne, Switzerland, September 30 - October 4, 2002, Lausanne, Switzerland, pp 853-858

[16] Martinelli A and Siegwart R., "Estimating the Odometry Error of a Mobile Robot during Navigation," accepted at the European Conference on Mobile Robots (ECMR 2003), Warsaw, Poland, September 4-6, 2003

[17] Papoulis A., Probability, Random Variables, and Stochastic Process McGRAW-HILL INTERNATIONAL EDITIONS, 1991

[18] Wang C.M., "Location estimation and uncertainty analysis for mobile robots," International Conference on Robotics and Automation, pp. 1231-1235, 1988.

[19] Zhejun F., Borenstein J., Wehe D., Koren Y., "Experimental evaluation of an Encoder Trailer for deadreckoning in tracked mobile robots ," IEEE International Symposium on Intelligent Control, pp. 571576, 1995. 